\title{
Cube is a Strict Local Maximizer for the Illumination Number
}

\author{
Galyna Livshyts ${ }^{1} \cdot$ Konstantin Tikhomirov $^{1}$
}

Received: 24 December 2017 / Revised: 3 May 2019 / Accepted: 13 July 2019 / Published online: 19 August 2019 (c) The Author(s) 2019

\begin{abstract}
It was conjectured by Levi, Hadwiger, Gohberg and Markus that the boundary of any convex body in $\mathbb{R}^{n}$ can be illuminated by at most $2^{n}$ light sources, and, moreover, $2^{n}-1$ light sources suffice unless the body is a parallelotope. We show that if a convex body is close to the cube in the Banach-Mazur metric, and it is not a parallelotope, then indeed $2^{n}-1$ light sources suffice to illuminate its boundary. Equivalently, any convex body sufficiently close to the cube, but not isometric to it, can be covered by $2^{n}-1$ smaller homothetic copies of itself.
\end{abstract}

Keywords Convex body · Illumination · Covering by homothetic copies

Mathematics Subject Classification 52A20 · 52C17

\section{Introduction}

The Levi-Hadwiger-Gohberg-Markus illumination conjecture [8,9] is a famous old question in discrete geometry, which has several equivalent formulations. The viewpoint we adopt in this note is due to Boltyanski (see, for example, $[1,4,6]$ ): Let $B$ be a convex body (i.e., compact convex set with non-empty interior) in $\mathbb{R}^{n}$ and let $\partial B$ denote its boundary. We say that a point $x \in \partial B$ is illuminated in direction $y \in \mathbb{R}^{n} \backslash\{0\}$ if there is a small positive $\varepsilon$ such that $x+\varepsilon y$ lies in the interior of $B$. Further, a collection $\left\{y^{1}, y^{2}, \ldots, y^{m}\right\}$ of non-zero vectors illuminates $B$ if for any $x \in \partial B$ there is $i=i(x) \leq m$ such that $x$ is illuminated in direction $y^{i}$. For any convex body $B$, denote by $\mathcal{I}(B)$ the cardinality of the smallest set of directions sufficient to illuminate all the points from $\partial B$. The number $\mathcal{I}(B)$ is called the illumination number of $B$.

Editor in Charge: János Pach

Galyna Livshyts

glivshyts6@math.gatech.edu

Konstantin Tikhomirov

konstantin.tikhomirov@math.gatech.edu

1 School of Mathematics, Georgia Institute of Technology, Atlanta, GA, USA 
Conjecture (Levi-Hadwiger-Gohberg-Markus) For any convex body $B$ in $\mathbb{R}^{n}$ we have $\mathcal{I}(B) \leq 2^{n}$, and equality holds if and only if $B$ is a parallelotope.

As of this writing, the best known general upper bound for the illumination number is due to Rogers [18]:

$$
\mathcal{I}(B) \leq(n \log n+n \log \log n+5 n) \frac{\operatorname{Vol}_{n}(B-B)}{\operatorname{Vol}_{n}(B)},
$$

where $B-B$ is the Minkowski sum of $B$ and $-B$ (called the difference body of $B$ ), and $\operatorname{Vol}_{n}(\cdot)$ is the Lebesgue volume in $\mathbb{R}^{n}$ (see, in particular, [4, Cor. 2.11] and [1, Cor. 3.4.2], as well as [14] where a "fully random" proof of Rogers' theorem is given). If $B$ is origin-symmetric then $\operatorname{Vol}_{n}(B-B)=2^{n} \operatorname{Vol}_{n}(B)$, whence $\mathcal{I}(B) \leq(n \log n+n \log \log n+5 n) 2^{n}$. For non-symmetric convex bodies, the relation $\operatorname{Vol}_{n}(B-B) \leq 4^{n} \operatorname{Vol}_{n}(B)$ due to Rogers and Shephard [19] implies that $\mathcal{I}(B) \leq(n \log n+n \log \log n+5 n) 4^{n}$.

The illumination conjecture has been solved (or almost solved) in some special cases. In particular, it is known that each origin-symmetric convex body in $\mathbb{R}^{3}$ can be illuminated by eight directions or fewer [12]. The conjecture holds true for so-called belt polytopes [15] and their generalization - the belt bodies [5]; for bodies of constant width [20] and, more generally, for the fat spindle bodies [2]; for dual cyclic polytopes [3]. Recently, it has been shown in [21] that unit balls of 1-symmetric normed spaces different from $\ell_{\infty}^{n}$ can be illuminated in less than $2^{n}$ directions. Finally, it is of interest to note that there exist convex bodies arbitrarily close to the Euclidean ball in the Banach-Mazur metric whose illumination number is exponential in the dimension [16]. Let us refer to [1, Chap. 3] and [4] for more information on the subject. Let us also mention a computer assisted approach to Hadwiger's conjecture [22].

The approach to the illumination problem that we consider in this note was inspired by the work [17] devoted to estimating the product of volumes of a convex body and its polar. In what follows, for any $p \in[1, \infty]$ we denote by $B_{p}^{n}$ the closed unit ball of the canonical $\|\cdot\|_{p}$-norm on $\mathbb{R}^{n}$. The Mahler conjecture, one of the central problems in convex geometry, asserts that for any origin-symmetric convex body $L$, the Mahler volume $\operatorname{Vol}_{n}(L) \cdot \operatorname{Vol}_{n}\left(L^{\circ}\right)$ is greater or equal to the Mahler volume of the cube $\operatorname{Vol}_{n}\left(B_{\infty}^{n}\right) \cdot \operatorname{Vol}_{n}\left(B_{1}^{n}\right)$, where $L^{\circ}$ denotes the polar body for $L$. We refer to [7] (see also [11]) for an "isomorphic solution" to this problem and related information. Very recently, this conjecture was verified in $\mathbb{R}^{3}$ in [10]. In [17] the Mahler conjecture was confirmed in every dimension in a small neighborhood of the cube and, moreover, it was shown that the cube is a strict local minimizer in the Banach-Mazur metric on the class of symmetric convex bodies. Let us recall that for any two (not necessarily centrally-symmetric) convex bodies $B$ and $L$ in $\mathbb{R}^{n}$, the Banach-Mazur distance $\mathrm{d}_{\mathrm{BM}}(B, L)$ between $B$ and $L$ is defined as the infimum of $\lambda \geq 1$ such that there is an invertible linear operator $T_{\lambda}: \mathbb{R}^{n} \rightarrow \mathbb{R}^{n}$ and two vectors $x_{\lambda}$ and $y_{\lambda}$ satisfying $B \subset T_{\lambda}(L)+x_{\lambda} \subset \lambda B+y_{\lambda}$. The main theorem of [17] asserts that for every positive integer $n$ there is $\delta(n)>0$ such that any origin-symmetric convex body $L$ with $1 \neq \mathrm{d}_{\mathrm{BM}}\left(L, B_{\infty}^{n}\right) \leq 1+\delta(n)$ satisfies $\operatorname{Vol}_{n}(L) \cdot \operatorname{Vol}_{n}\left(L^{\circ}\right)>\operatorname{Vol}_{n}\left(B_{\infty}^{n}\right) \cdot \operatorname{Vol}_{n}\left(B_{1}^{n}\right)$.

In this paper, we apply the "local" viewpoint of [17] in the context of the illumination conjecture. The nature of the illumination problem is very different from the Mahler 
conjecture discussed above; the most obvious distinction coming from the lack of continuity. Whereas the volume is stable with respect to small perturbations of a convex body, the structure of its boundary - the layout of the extreme points, and the combinatorial structure (in case of polytopes) — can change significantly even with arbitrarily small perturbations. It becomes an interesting feature that certain "discretegeometric" properties related to the illumination (which we will mention later), remain stable in a small neighborhood of the cube. The main non-technical result of this paper is the following theorem.

Theorem 1.1 For any $n \geq 3$ there is a $\delta=\delta(n)>0$ with the following property: Let $B$ be a convex body in $\mathbb{R}^{n}$ and assume that $1 \neq \mathrm{d}_{\mathrm{BM}}\left(B, B_{\infty}^{n}\right) \leq 1+\delta$. Then $B$ can be illuminated with $2^{n}-1$ directions or fewer.

It is well known that the illumination number of $B$ can be equivalently defined as the least number of translates of the interior of $B$ needed to cover $B$ (see, in particular [6], [1, Chap. 3], [4]). In this sense, it becomes a simple observation that any convex body with a sufficiently small Banach-Mazur distance to the cube can be illuminated by $2^{n}$ directions or fewer (in fact, more general statements are known; see [4, Sect. 4] and references therein). The non-triviality of the above theorem consists in proving that strictly less than $2^{n}$ light sources suffice.

The construction of an illuminating set for $B$ involves a careful study of its geometry. Naturally, we consider the canonical illuminating set of the cube- the set of all sign vectors - as the starting point. Next, we determine which pair of adjacent illuminating directions can be "glued together" to form a single light source. The procedure is completed by repositioning several of the "canonical" light sources in a special way. Thus, we show that for any body $B$ close to the cube in the Banach-Mazur metric but not isometric to the cube, we can find a distinguished pair of boundary points of $B$ illuminated by the same light source, and complete the illumination of the entire boundary of $B$ by adding $2^{n}-2$ light sources in "standard" or "almost standard" positions. Interestingly, existence of this distinguished pair is a feature of all convex sets sufficiently close to the cube (but not the cube itself). We will give a complete description of this strategy later.

The dependency of the quantity $\delta=\delta(n)$ of the main theorem on the dimension $n$ is not explicit as we use continuity arguments to establish certain properties of convex sets close to the cube. In particular, it is an interesting question how large $\delta$ can be made by replacing the implicit estimates with more precise computations (while keeping the same proof structure). We anticipate that this question will be addressed in our future works.

Remark 1.2 We would like to point it out that our estimate is sharp in the sense that for every $n \geq 2$ and for every $\varepsilon \in(0,1)$ there exists a convex body $B$ in $\mathbb{R}^{n}$ with $\mathrm{d}_{\mathrm{BM}}\left(B, B_{\infty}^{n}\right) \leq 1+\varepsilon$ and such that the illumination number of $B$ is exactly $2^{n}-1$. We consider the following construction.

Let $v:=(1,1, \ldots, 1), v^{\prime}:=(-1,1,1, \ldots, 1)$ and, for $\varepsilon \in(0,1)$, let $\tilde{v}:=$ $(1-\varepsilon, 1,1, \ldots, 1)$. Set

$$
B:=\operatorname{conv}\left(\{-1,1\}^{n} \backslash v, \tilde{v}\right),
$$


where "conv $(S)$ " denotes the convex hull of a set $S$, i.e., the smallest convex set containing $S$. Note that $\mathrm{d}_{\mathrm{BM}}\left(B, B_{\infty}^{n}\right) \leq 1 /(1-\varepsilon)$.

For each vertex $w$ of $B$, consider the set of vectors which illuminate $w$ as a boundary point of $B$ :

$$
\eta(B, w):=\left\{x \in \mathbb{R}^{n}: \exists \delta>0 \text { such that } w+\delta x \text { is in the interior of } B\right\} .
$$

Note that for all the vertices $w$ of $B$ which have at least two negative coordinates, as well as for $w=v^{\prime}$, one has $\eta(B, w)=\left\{x \in \mathbb{R}^{n}: \operatorname{sign}\left(x_{i}\right)=-\operatorname{sign}\left(w_{i}\right)\right.$ for all $i \leq$ $n\}$. Moreover, for all vertices $w$ of $B$ with exactly one negative coordinate one has $\eta(B, w) \subset\left\{x \in \mathbb{R}^{n}: \operatorname{sign}\left(x_{i}\right)=-\operatorname{sign}\left(w_{i}\right)\right.$ for all $\left.i \leq n\right\}$. This implies that the illuminating sets for $\{-1,1\}^{n} \backslash v$ are pairwise disjoint, whence $\mathcal{I}(B) \geq 2^{n}-1$.

\section{Notation and Preliminaries}

Given a positive integer number $n,[n]$ is the set $\{1,2, \ldots, n\}$. We denote by $e_{1}, e_{2}, \ldots, e_{n}$ the standard basis in $\mathbb{R}^{n}$ and by $\langle\cdot, \cdot\rangle$ - the canonical inner product in $\mathbb{R}^{n}$. Given any $1 \leq p \leq \infty$, let $\|\cdot\|_{p}$ be the $\ell_{p}^{n}$-norm on $\mathbb{R}^{n}$, i.e.,

$$
\left\|\left(x_{1}, x_{2}, \ldots, x_{n}\right)\right\|_{p}:=\left(\sum_{i=1}^{n}\left|x_{i}\right|^{p}\right)^{1 / p} \text { and }\left\|\left(x_{1}, x_{2}, \ldots, x_{n}\right)\right\|_{\infty}:=\max _{i \leq n}\left|x_{i}\right|
$$

The unit ball of the $\ell_{p}^{n}$-norm is denoted by $B_{p}^{n}$. By I we denote the identity operator in $\mathbb{R}^{n}$ (the dimension $n$ will always be clear from the context). Further, given a linear operator $T: \mathbb{R}^{n} \rightarrow \mathbb{R}^{n}$, let $\|T\|:=\|T\|_{2 \rightarrow 2}$ stand for the spectral norm of $T$ (i.e., its largest singular value), and, more generally, for any two numbers $1 \leq p, q \leq \infty$, let $\|T\|_{p \rightarrow q}$ be the operator norm of $T$ considered as a mapping from $\ell_{p}^{n}$ to $\ell_{q}^{n}$. Thus,

$$
\|T\|_{p \rightarrow q}:=\sup _{\|x\|_{p}=1}\|T x\|_{q} .
$$

In view of standard comparison inequalities for $\ell_{p}^{n}$-norms, we have

$$
\|T\|_{p \rightarrow q} \leq n^{2}\|T\|_{p^{\prime} \rightarrow q^{\prime}} \quad \text { for all } 1 \leq p, q, p^{\prime}, q^{\prime} \leq \infty .
$$

Given a cone $K \subset \mathbb{R}^{n}$ with the vertex at the origin, we define the solid angle $\sigma(K)$ as

$$
\sigma(K):=\frac{\operatorname{Vol}_{n}\left(K \cap B_{2}^{n}\right)}{\operatorname{Vol}_{n}\left(B_{2}^{n}\right)}
$$

Let $B$ be a convex body in $\mathbb{R}^{n}$. Recall that the Gauss map $v_{B}: \partial B \rightarrow \mathbb{S}^{n-1}$ maps each point $x \in \partial B$ to the collection of outer unit normals to supporting hyperplanes at $x$. The Gauss image $v(B, x)$ of $x \in \partial B$ is the convex cone given by $\{\lambda y: y \in$ 
$\left.v_{B}(x), \lambda \geq 0\right\}$. Further, each point $x \in \partial B$ can be associated with another convex cone, the illuminating $\operatorname{set} \eta(B, x)$ which comprises all non-zero directions illuminating $x$. Note that $v(B, x)$ and the closure of $\eta(B, x)$ are polar cones, that is,

$$
v(B, x)=\left\{y \in \mathbb{R}^{n}:\langle y, z\rangle \leq 0 \text { for all } z \in \eta(B, x)\right\}
$$

Given a point $x \in \partial B$, denote by $\sigma(B, x)$ the solid angle of the cone $\eta(B, x)$.

The next simple lemma will be useful; we give a proof for the reader's convenience.

Lemma 2.1 For any $n>1$ and $\beta>0$ there is $r_{2.1}=r_{2.1}(n, \beta)>0$ with the following property: Let $P$ be a non-degenerate parallelotope in $\mathbb{R}^{n}$ such that for each vertex $v$ of the standard cube $B_{\infty}^{n}$ there is a vertex $v^{\prime}$ of $P$ satisfying $v^{\prime}-v \in r_{2.1} B_{\infty}^{n}$. Then there is an invertible linear operator $T$ in $\mathbb{R}^{n}$ and a vector $y$ such that $B_{\infty}^{n}=T(P)+y$ and $\|T-\mathrm{I}\|,\left\|T^{-1}-\mathrm{I}\right\| \leq \beta$.

Proof Fix a small $\beta>0$ and define $\beta^{\prime}:=\beta / n^{2}$ and $r=r_{2.1}:=\beta^{\prime} / 4$. Let $P$ be a parallelotope in $\mathbb{R}^{n}$ satisfying the conditions of the lemma. First, observe that $(1-r) B_{\infty}^{n} \subset P$. Indeed, otherwise there would exist a vertex $v$ of $(1-r) B_{\infty}^{n}$ and an affine hyperplane $H$ passing through $v$ and not intersecting $P$. On the other hand, one could always find a pair of opposite vertices of $B_{\infty}^{n}$ lying in different half-spaces (determined by $H$ ) and such that the $\ell_{\infty}^{n}$-distance of either vertex to $H$ is greater than $r$. This would contradict the assumption that every vertex of the cube can be $r$-approximated by a point in $P$ in the $\ell_{\infty}^{n}$-metric.

For each $v \in\{-1,1\}^{n}$ let $f(v)$ be the (unique) vertex of $P$ satisfying $\| v-$ $f(v) \|_{\infty} \leq r$. Let $S$ be the set of all vertices of $B_{\infty}^{n}$ adjacent to $(1,1, \ldots, 1)$. Note that the $n$ vectors $\{f(1,1, \ldots, 1)-f(v)\}_{v \in S}$ are linearly independent whence there is a unique linear operator $T$ and a vector $y \in \mathbb{R}^{n}$ such that $(1,1, \ldots, 1)=$ $T(f(1,1, \ldots, 1))+y$ and $v=T(f(v))+y(v \in S)$. Note that necessarily $T(P)+y=$ $B_{\infty}^{n}$, and, moreover, $f$ is the restriction of $T^{-1}(\cdot)-T^{-1}(y)$ to $\{-1,1\}^{n}$. Together with the inclusion $(1-r) B_{\infty}^{n} \subset P$, this gives $(1-r) T\left(B_{\infty}^{n}\right) \subset B_{\infty}^{n}-y$, whence, by the symmetry of $T\left(B_{\infty}^{n}\right),(1-r) T\left(B_{\infty}^{n}\right) \subset B_{\infty}^{n}$, and $\|T\|_{\infty \rightarrow \infty} \leq(1-r)^{-1}$.

By linearity of $T$, we have

$$
0=2^{-n} \sum_{v \in\{-1,1\}^{n}} T^{-1}(v)=T^{-1}(y)+2^{-n} \sum_{v \in\{-1,1\}^{n}} f(v),
$$

whence

$$
\begin{aligned}
\left\|T^{-1}(y)\right\|_{\infty} & =2^{-n}\left\|\sum_{v \in\{-1,1\}^{n}} f(v)\right\|_{\infty}=2^{-n}\left\|\sum_{v \in\{-1,1\}^{n}}(v-f(v))\right\|_{\infty} \\
& \leq 2^{-n} \sum_{v \in\{-1,1\}^{n}}\|v-f(v)\|_{\infty} \leq r .
\end{aligned}
$$


Next, elementary convexity properties and the bound $\|T\|_{\infty \rightarrow \infty} \leq(1-r)^{-1}<2$ imply

$$
\begin{aligned}
\|T-\mathrm{I}\|_{\infty \rightarrow \infty} & =\max _{v \in\{-1,1\}^{n}}\|T v-v\|_{\infty} \\
& <2 \max _{v \in\{-1,1\}^{n}}\left\|v-f(v)-T^{-1}(y)\right\|_{\infty} \leq 4 r=\beta^{\prime} .
\end{aligned}
$$

Further,

$$
\begin{aligned}
\left\|T^{-1}-\mathrm{I}\right\|_{\infty \rightarrow \infty} & =\max _{v \in\{-1,1\}^{n}}\left\|T^{-1} v-v\right\|_{\infty} \\
& =\max _{v \in\{-1,1\}^{n}}\left\|f(v)-v+T^{-1}(y)\right\|_{\infty} \leq 2 r \leq \beta^{\prime}
\end{aligned}
$$

Finally, note that in view of (1) we get from the above that $\|T-\mathrm{I}\| \leq \beta^{\prime} n^{2}=\beta$ and $\left\|T^{-1}-\mathrm{I}\right\| \leq \beta^{\prime} n^{2}=\beta$. The result follows.

\section{High-Level Structure of the Proof}

In this section, we give the proof of the main theorem assuming several properties of convex bodies close to the cube (they are stated as lemmas). The proofs of the lemmas which constitute the technical part of the paper, are deferred to the next section. In the proof of the theorem, we work with quantities depending on various parameters or other functions. For example, by writing $\beta=\beta(n, \alpha)$ we introduce $\beta$ as a function of two variables $n$ and $\alpha$. To make referencing easier, each function introduced within a lemma is written with the number of that lemma as a subscript.

The invariance of the illumination number under affine transformations allows us to restrict our analysis to the class of convex bodies $B \subset \mathbb{R}^{n}$ such that

$$
B_{\infty}^{n} \subset B \subset \mathrm{d}_{\mathrm{BM}}\left(B, B_{\infty}^{n}\right) B_{\infty}^{n}+y
$$

for some vector $y=y(B) \in \mathbb{R}^{n}$. We say that a body $B$ satisfying ( $\star$ ) is in $a \star$-position. Note that the $\star$-position is not uniquely defined in general. It is obvious that any convex body $B$ in a $\star-$ position satisfies

$$
B \subset\left(2 \mathrm{~d}_{\mathrm{BM}}\left(B, B_{\infty}^{n}\right)-1\right) B_{\infty}^{n}
$$

Now, assume we have a convex body $B$ in a $\star$-position, with a very small BanachMazur distance to the cube (but not the cube itself). How could we construct an illuminating set for $B$ of cardinality $2^{n}-1$ ? It is natural to start with the standard illuminating set for the cube, i.e., the set $\{-1,1\}^{n}$, transform it in some way and remove one direction. One may note that simply excluding one direction from $\{-1,1\}^{n}$, without changing the remaining directions is not sufficient. Indeed, consider a convex polygon $P$ in $\mathbb{R}^{2}$ with eight vertices $\pm(1+\varepsilon, 1-\varepsilon), \pm(1-\varepsilon, 1+\varepsilon), \pm(-1+\varepsilon, 1+$ $\varepsilon), \pm(-1-\varepsilon, 1-\varepsilon)$, where $\varepsilon>0$ is small enough. It can be checked that $P$ is in a $\star$-position, and that any proper subset of $\{-1,1\}^{2}$ does not illuminate $P$. 
Another natural approach is to replace a pair of adjacent illumination directions with a single vector. That is, given a convex body $B$ in $\mathbb{R}^{n}$ in a $\star$-position and with a small distance to cube, construct an illumination set of the form

$$
\left(\{-1,1\}^{n} \backslash\left\{-v,-v^{\prime}\right\}\right) \cup\{\mathbf{w}\}
$$

where $\left\{-v,-v^{\prime}\right\}$ is some pair of adjacent directions from $\{-1,1\}^{n}$ and $\mathbf{w}$ is some nonzero vector in $\mathbb{R}^{n}$. It can be checked directly that this method fails for the polygon $P$ constructed above, but, by slightly repositioning the canonical illumination directions, it is possible to get an illuminating set for $P$ (say, take the set $\{(1,-1.1),(1,1.1)\} \cup\{\mathbf{w}\}$ with $\mathbf{w}=(-1,0))$. It turns out that this approach can be generalized to convex bodies in higher dimensions; thus, our construction in Theorem 1.1 resembles (3) but is somewhat more technical. Let us give a formal definition.

For any $n \geq 3$ and any choice of parameters $\varepsilon, \theta \in(0,1)$, let us denote by $\mathcal{C}_{n}(\varepsilon, \theta)$ the collection of all subsets $S \subset \mathbb{R}^{n}$ of cardinality $2^{n}-1$ such that there is a pair $v=v(S), v^{\prime}=v^{\prime}(S)$ of adjacent vertices of the standard cube $B_{\infty}^{n}$ satisfying the following three conditions:

- For any vertex $w \in\{-1,1\}^{n}$ adjacent neither to $v$ nor to $v^{\prime}, S$ contains a vector in the set $-w+\varepsilon B_{\infty}^{n}$.

- For any vertex $w=\left(w_{1}, w_{2}, \ldots, w_{n}\right) \in\{-1,1\}^{n} \backslash\left\{v, v^{\prime}\right\}$ adjacent either to $v$ or $v^{\prime}, S$ contains a vector in

$$
-\sum_{j: j \neq i} w_{j} e_{j}-\theta w_{i} e_{i}+\varepsilon B_{\infty}^{n}
$$

where $i=i(w)$ is the unique index in $[n]$ such that $v_{i}=v_{i}^{\prime} \neq w_{i}$.

- $S$ contains a vector $\mathbf{w}=\left(\mathbf{w}_{1}, \mathbf{w}_{2}, \ldots, \mathbf{w}_{n}\right)$ in the parallelepiped

$$
-\prod_{j=1}^{n}\left(\frac{v_{j}+v_{j}^{\prime}}{2} \cdot[\theta, 1]\right)+\varepsilon B_{\infty}^{n} .
$$

Note here that if $\ell \in[n]$ is the unique index with $v_{\ell}=-v_{\ell}^{\prime}$, we get $\left|\mathbf{w}_{\ell}\right| \leq \varepsilon$.

We will call $\mathbf{w}$ the distinguished direction of the set $S$, and $v, v^{\prime}$ the distinguished vertices of the cube w.r.t. set $S$. Roughly speaking, each set in $\mathcal{C}_{n}(\varepsilon, \theta)$ is constructed by taking a standard illuminating set $\{-1,1\}^{n}$ of $B_{\infty}^{n}$, glueing together a pair of adjacent illuminating directions and then perturbing the collection in a special way. The principal difference of the above construction from (3) is that we reposition all illuminating directions adjacent to either $-v$ or $-v^{\prime}$ by moving them "closer" to $-v,-v^{\prime}$ (the reader may wish to compare this strategy to our illumination of the polytope $P$ in the above example). The rest of the illuminating directions, disregarding a small perturbation, remain unchanged (i.e., are essentially sign vectors).

Observe that for any $0<\varepsilon<\varepsilon^{\prime}$ and $\theta>0$ we have $\mathcal{C}_{n}(\varepsilon, \theta) \subset \mathcal{C}_{n}\left(\varepsilon^{\prime}, \theta\right)$. The definition of the class $\mathcal{C}_{n}(\varepsilon, \theta)$, being somewhat technical, is designed to be "stable" with respect to linear transformations close to the identity: 
Lemma 3.1 For any $n>2, \varepsilon, \theta \in(0,1)$ and $\alpha>0$ there is $\beta_{3 . l}=\beta_{3, l}(n, \alpha)>0$ with the following property: whenever $S \in \mathcal{C}_{n}(\varepsilon, \theta)$ and $T$ is a linear operator in $\mathbb{R}^{n}$ satisfying $\|T-\mathrm{I}\| \leq \beta_{3.1}$, we have $T(S) \in \mathcal{C}_{n}(\varepsilon+\alpha, \theta)$.

Our goal is to show that if $B \subset \mathbb{R}^{n}$ is a convex body in a $\star$-position which is very close to the cube, yet distinct from it, then $B$ can be illuminated by a collection $S \in \mathcal{C}_{n}(\varepsilon, \theta)$ for some appropriately chosen parameters $\varepsilon, \theta$. Both parameters will be taken sufficiently small, but, importantly, $\varepsilon$ shall be much smaller than $\theta$.

A crucial notion that will help us to study illumination by directions from $\mathcal{C}_{n}(\varepsilon, \theta)$ is that of pseudo-vertices. Let $r \in(0,1)$ be a parameter and let $B$ be a body in $\mathbb{R}^{n}$ in a $\star$-position with $\mathrm{d}_{\mathrm{BM}}\left(B, B_{\infty}^{n}\right) \leq 1+r / 2$. Note that, in view of (2), for any vertex $v$ of the standard cube $B_{\infty}^{n}$, the set $r B_{\infty}^{n}+v$ intersects with the boundary of $B$. Given any vertex $v$ of $B_{\infty}^{n}$, we say that a point $p \in\left(r B_{\infty}^{n}+v\right) \cap \partial B$ is an $(r)$-pseudo-vertex of $B$ if $p$ has minimal solid angle $\sigma(B, p)$ among all points in $\left(r B_{\infty}^{n}+v\right) \cap \partial B$. For all admissible $r$, the set $B$ has at least $2^{n}(r)$-pseudo-vertices, but may have more (in fact, uncountably many if the boundary of $B$ is smooth). Further, we say that a collection of $2^{n}$ points $\mathcal{V} \subset \mathbb{R}^{n}$ is a proper set of (r)-pseudo-vertices of $B$ if for any vertex $v$ of $B_{\infty}^{n}, \mathcal{V}$ contains exactly one $(r)$-pseudo-vertex $p(v) \in\left(r B_{\infty}^{n}+v\right) \cap \partial B$. In particular, if $B=B_{\infty}^{n}$ then the proper set of (r)-pseudo-vertices (for any admissible value of $r$ ) coincides with the set of the regular vertices of the cube. In general, $\mathcal{V}$ may be not uniquely defined.

It is easy to see that any point $x$ on the boundary of the standard cube such that $\sigma\left(B_{\infty}^{n}, x\right)<2^{-n+1}$, must be one of its vertices. Below we state a weaker relative of this property for convex bodies sufficiently close to the cube and their pseudo-vertices.

Lemma 3.2 For each $n>2$ there are $\eta_{3.2}=\eta_{3.2}(n) \in(0,1), \kappa_{3.2}=\kappa_{3.2}(n)>0$ with the following property. Let $0<\eta \leq \eta_{3.2}$. Then there is $\delta_{3.2}=\delta_{3.2}(n, \eta) \in(0, \eta / 2)$ such that for any convex body $B$ in $\mathbb{R}^{n}$ in $a \star$-position, with $\mathrm{d}_{\mathrm{BM}}\left(B, B_{\infty}^{n}\right) \leq 1+\delta_{3.2}$, and for any point $x \in \partial B$ with $\sigma(B, x) \leq\left(1+\kappa_{3.2}\right) \cdot 2^{-n}$, there is a vertex $v$ of the standard cube $B_{\infty}^{n}$ such that $x$ is the unique $(\eta)$-pseudo-vertex of $B$ in $\left(\eta B_{\infty}^{n}+v\right) \cap \partial B$.

Thus, we can detect a pseudo-vertex if its solid angle is less than certain critical value. The following lemma provides a connection between this property of a boundary point and the illumination of $B$, and, together with Lemma 3.6, comprises the most technical part of the paper:

Lemma 3.3 For any $n>2, \kappa>0$ there is $\theta_{3,3}(n, \kappa) \in(0,1)$ with the following property. For any $0<\theta \leq \theta_{3.3}$ there are $\delta_{3.3}=\delta_{3.3}(n, \kappa, \theta)>0$ and $\varepsilon_{3.3}=\varepsilon_{3.3}(n, \kappa, \theta) \in$ $(0,1)$ such that for any convex body $B$ in a $a$-position with $\mathrm{d}_{\mathrm{BM}}\left(B, B_{\infty}^{n}\right) \leq 1+\delta_{3.3}$ and any element $S \in \mathcal{C}_{n}\left(\varepsilon_{3.3}, \theta\right)$ we have that every point $x \in \partial B$ not illuminated by $S$ satisfies

$$
\sigma(B, x) \leq(1+\kappa) \cdot 2^{-n}
$$

The following two lemmas are the core of our argument:

Lemma 3.4 Let $n>2$ and let $\varepsilon, \theta \in(0,1)$ be any numbers. Let $B$ be a convex body in $\mathbb{R}^{n}$ such that $\{-1,1\}^{n} \subset \partial B$ and $B \neq B_{\infty}^{n}$. Then there is a set $S \in \mathcal{C}_{n}(\varepsilon, \theta)$ which illuminates every point in $\{-1,1\}^{n}$ (viewed as boundary points for $B$ ). 
Remark 3.5 Note that in the above lemma we illuminate only a subset of the boundary and not the entire body.

Lemma 3.6 For any $n>2$ and $\varepsilon \in(0,1)$ there is $\theta_{3.6}=\theta_{3.6}(n)$ with the following property. Let $0<\theta \leq \theta_{3.6}$. Then there is $r_{3.6}=r_{3.6}(n, \varepsilon, \theta) \in(0,1 / 2)$ such that for any convex polytope $P$ with $2^{n}$ vertices and $\mathrm{d}_{\mathrm{BM}}\left(P, B_{\infty}^{n}\right) \neq 1$ so that for each $v \in\{-1,1\}^{n}$ there is a vertex $v^{\prime}$ of $P$ with $v^{\prime}-v \in r_{3.6} B_{\infty}^{n}$, we have that $P$ is illuminated by some set $S \in \mathcal{C}_{n}(\varepsilon, \theta)$.

Remark 3.7 It is crucial that the parameter $\theta_{3.6}$ in the above lemma depends only on $n$ and not on $\varepsilon$.

The above statements allow to complete the proof of the main theorem:

Proof of Theorem 1.1 Let us start by defining parameters. We fix any $n>2$ and set

$$
\begin{aligned}
& \theta:=\min \left(\theta_{3.6}(n), \theta_{3.3}\left(n, \kappa_{3.2}(n)\right)\right), \quad \varepsilon:=\varepsilon_{3.3}\left(n, \kappa_{3.2}(n), \theta\right), \quad \beta:=\beta_{3.1}(n, \varepsilon / 2), \\
& r:=\min \left(r_{3.6}(n, \varepsilon, \theta), r_{2.1}(n, \beta), \eta_{3.2}(n)\right), \quad \delta:=\min \left(\delta_{3.3}\left(n, \kappa_{3.2}(n), \theta\right), \delta_{3.2}(n, r)\right) .
\end{aligned}
$$

Consider a convex body $B$ in $\mathbb{R}^{n}$ with $1 \neq \mathrm{d}_{\mathrm{BM}}\left(B, B_{\infty}^{n}\right) \leq 1+\delta$. Assume that $B$ is in a $\star$-position. Let $\mathcal{V}$ be a proper set of $(r)$-pseudo-vertices of $B$ (note that $\delta \leq r / 2$ so $\mathcal{V}$ exists).

As the first step, we show that there is a set of directions $S \in \mathcal{C}_{n}(\varepsilon, \theta)$ which illuminates $\operatorname{conv}(\mathcal{V})$. Indeed, if $\operatorname{conv}(\mathcal{V})$ is not a parallelotope then the assertion follows from Lemma 3.6 and our choice of parameters. Otherwise, if $\mathrm{d}_{\mathrm{BM}}\left(\operatorname{conv}(\mathcal{V}), B_{\infty}^{n}\right)=1$ then, in view of Lemma 2.1, there is a linear operator $T$ and a vector $y$ in $\mathbb{R}^{n}$ such that $\|T-\mathrm{I}\| \leq \beta,\left\|T^{-1}-\mathrm{I}\right\| \leq \beta$ and $T(\operatorname{conv}(\mathcal{V}))+y=B_{\infty}^{n}$. By Lemma 3.4, we can find a set $S^{\prime} \in \mathcal{C}_{n}(\varepsilon / 2, \theta)$ which illuminates points in $\{-1,1\}^{n}$ considered as boundary points of $T(B)+y$. Hence, $T^{-1}\left(S^{\prime}\right)$ illuminates $\operatorname{conv}(\mathcal{V})$ (again, viewed as a subset of the boundary of $B)$. Due to the assumptions on $T$ and Lemma 3.1, $S:=T^{-1}\left(S^{\prime}\right)$ belongs to $\mathcal{C}_{n}(\varepsilon, \theta)$.

Now, having constructed $S$, assume that there is a point $x \in \partial B$ which is not illuminated by $S$. Then, in view of Lemma 3.3 and our choice of parameters, we have $\sigma(B, x) \leq\left(1+\kappa_{3.2}(n)\right) \cdot 2^{-n}$. But then, by Lemma 3.2 (applied with $\left.\eta:=r\right), x$ is the unique $(r)$-pseudo-vertex in $\left(r B_{\infty}^{n}+v\right) \cap \partial B$ for some vertex $v$ of the standard cube $B_{\infty}^{n}$. Hence, $x$ must belong to $\mathcal{V}$ leading to contradiction. Thus, $B$ is entirely illuminated in $2^{n}-1$ directions.

\section{Proofs of Lemmas 3.1-3.6}

\subsection{Proof of Lemma 3.1}

Fix $n>2$ and parameters $\varepsilon, \theta \in(0,1), \alpha>0$. Instead of the spectral norm $\|\cdot\|$, it will be convenient to consider $\|\cdot\|_{\infty \rightarrow \infty}$ (this makes no difference since we allow the parameter $\beta_{3.1}$ to depend on $n$ ). Set $\beta:=\alpha / 2$. Suppose $T$ is a linear operator satisfying $\|T-\mathrm{I}\|_{\infty \rightarrow \infty} \leq \beta$. 
Take a vertex $w$ of the standard cube and let $p \in-w+\varepsilon B_{\infty}^{n}$. Observe that $\|p+w\|_{\infty} \leq \varepsilon$ and $\|p\|_{\infty} \leq 1+\varepsilon$. Thus

$$
\begin{aligned}
\|T p+w\|_{\infty} & \leq\|T p-p\|_{\infty}+\|p+w\|_{\infty} \leq\|T-\mathrm{I}\|_{\infty \rightarrow \infty}\|p\|_{\infty}+\|p+w\|_{\infty} \\
& \leq \beta(1+\varepsilon)+\varepsilon \leq \alpha+\varepsilon,
\end{aligned}
$$

and therefore $T p \in-w+(\alpha+\varepsilon) B_{\infty}^{n}$.

In the same manner, given any $i \leq n$ and any $p^{\prime} \in-\sum_{j: j \neq i} w_{j} e_{j}-\theta w_{i} e_{i}+\varepsilon B_{\infty}^{n}$, we have

$$
\left\|T p^{\prime}+\left(\sum_{j: j \neq i} w_{j} e_{j}+\theta w_{i} e_{i}\right)\right\|_{\infty} \leq \alpha+\varepsilon
$$

Finally, for any two adjacent vertices $v, v^{\prime}$ of the standard cube and for any

$$
p^{\prime \prime} \in-\prod_{j=1}^{n}\left(\frac{v_{j}+v_{j}^{\prime}}{2} \cdot[\theta, 1]\right)+\varepsilon B_{\infty}^{n},
$$

we have

$$
T p^{\prime \prime} \in-\prod_{j=1}^{n}\left(\frac{v_{j}+v_{j}^{\prime}}{2} \cdot[\theta, 1]\right)+(\alpha+\varepsilon) B_{\infty}^{n} .
$$

Together with the definition of the classes $\mathcal{C}_{n}(\varepsilon, \theta)$, this implies the result.

\subsection{Proof of Lemma 3.2}

We will prove the following two claims.

Claim 4.1 For any $n>2$ and $\eta>0$ there is $\delta_{0}(n, \eta)>0$ with the following property. Let $B$ be a convex body in $\mathbb{R}^{n}$ with $B_{\infty}^{n} \subset B \subset\left(1+\delta_{0}\right) B_{\infty}^{n}$, and let $x$ be a point on the boundary of $B$ such that $\|x-v\|_{\infty} \geq \eta$ for all $v \in\{-1,1\}^{n}$. Then necessarily $\sigma(B, x) \geq 1.5 \cdot 2^{-n}$.

Claim 4.2 For any $n>2$ there are positive $\eta^{\prime}=\eta^{\prime}(n)<1$ and $\kappa^{\prime}=\kappa^{\prime}(n)<0.5$ with the following property. Let $B$ be a convex body in $\mathbb{R}^{n}$ satisfying $B_{\infty}^{n} \subset B$, let $v$ be any vertex of $B_{\infty}^{n}$ and let $x, y$ be two distinct points in $\partial B \cap\left(v+\eta^{\prime} B_{\infty}^{n}\right)$. Then necessarily $\max (\sigma(x), \sigma(y))>\left(1+\kappa^{\prime}\right) 2^{-n}$.

Now, it is not difficult to verify that the claims imply the assertion of the lemma. Indeed, fix any $0<\eta \leq \eta_{3.2}:=\eta^{\prime}(n)$ and set $\delta_{3.2}:=\min \left(\delta_{0}(n, \eta), \eta\right) / 2$. Let $B$ be a convex body in $\mathbb{R}^{n}$ in a $\star$-position, with $\mathrm{d}_{\mathrm{BM}}\left(B, B_{\infty}^{n}\right) \leq 1+\delta_{3.2}$. Observe that, in view of (2), we have $B \subset\left(1+2 \delta_{3.2}\right) B_{\infty}^{n}$. Let $x$ be a point on the boundary of $B$ such that $\sigma(B, x) \leq\left(1+\kappa_{3.2}\right) 2^{-n}$, with $\kappa_{3.2}:=\kappa^{\prime}(n)$. In view of Claim 4.1, we have $\|x-v\|_{\infty} \leq \eta$ for some $v \in\{-1,1\}^{n}$. At the same time, for any other point 
$y \in \partial B \cap\left(v+\eta B_{\infty}^{n}\right)$ we have, in view of Claim 4.2, $\sigma(B, y)>\left(1+\kappa_{3.2}\right) 2^{-n} \geq \sigma(B, x)$. In other words, $x$ is the (unique) minimizer for $\sigma(B, \cdot)$ in $\partial B \cap\left(v+\eta B_{\infty}^{n}\right)$. The lemma follows. Now, we prove the claims.

Proof of Claim 4.1 For any point $x \in \mathbb{R}^{n} \backslash \operatorname{Int}\left(B_{\infty}^{n}\right)$, define a convex cone $K_{x}:=$ $\left\{t(z-x): z \in B_{\infty}^{n}, t \geq 0\right\}$, and define a function $f(x): \mathbb{R}^{n} \backslash \operatorname{Int}\left(B_{\infty}^{n}\right) \rightarrow \mathbb{R}$ by $f(x):=\sigma\left(K_{x}, x\right)$. Observe that $f(x)$ is lower semi-continuous, that is, for any sequence $\left(x^{m}\right)_{m \geq 1}$ in $\mathbb{R}^{n} \backslash \operatorname{Int}\left(B_{\infty}^{n}\right)$ converging to a point $x$ we have $f(x) \leq$ $\liminf _{m \rightarrow \infty} f\left(x^{m}\right)$. Now, for any $\delta>0$ and $0<\eta<1$ consider the set

$$
A(\eta, \delta):=\left((1+\delta) B_{\infty}^{n} \backslash \operatorname{Int}\left(B_{\infty}^{n}\right)\right) \cap\left\{y \in \mathbb{R}^{n}:\|y-v\|_{\infty} \geq \eta \text { for all } v \in\{-1,1\}^{n}\right\}
$$

In other words, $A(\eta, \delta)$ is the set of points in the closed thin shell between the boundaries of cubes $B_{\infty}^{n}$ and $(1+\delta) B_{\infty}^{n}$, with $\ell_{\infty}^{n}$-distance to $\{-1,1\}^{n}$ at least $\eta$. Clearly, for any fixed $0<\eta<1$ the lower semi-continuity of $f(x)$ implies that

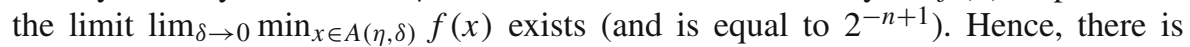
$\delta_{0}=\delta_{0}(\eta)>0$ with $\min _{x \in A\left(\eta, \delta_{0}\right)} f(x) \geq 1.5 \cdot 2^{-n}$. Now, for any convex body $B$ with $B_{\infty}^{n} \subset B \subset\left(1+\delta_{0}\right) B_{\infty}^{n}$ and $x \in \partial B$ with $\|x-v\|_{\infty} \geq \eta$ for all $v \in\{-1,1\}^{n}$, we have $x \in A\left(\eta, \delta_{0}\right)$, whence $\sigma(B, x) \geq f(x) \geq \min _{y \in A\left(\eta, \delta_{0}\right)} f(y) \geq 1.5 \cdot 2^{-n}$. The statement follows.

Proof of Claim 4.2 Let us make a few preliminary observations. First, the Euclidean distance from the point $p=(1 / \sqrt{n}, 1 / \sqrt{n}, \ldots, 1 / \sqrt{n})$ to each coordinate vector $e_{i}$ is

$$
\left\|p-e_{i}\right\|_{2}=\sqrt{2-\frac{2}{\sqrt{n}}} .
$$

On the other hand, given two opposite points $p_{1}$ and $p_{2}=-p_{1}$ on the unit sphere $\mathbb{S}^{n-1}$, we have

$$
\left\|p-p_{1}\right\|_{2}^{2}+\left\|p-p_{2}\right\|_{2}^{2}=4
$$

Therefore,

$$
\max \left(\left\|p-p_{1}\right\|_{2},\left\|p-p_{2}\right\|_{2}\right) \geq \sqrt{2}>\left\|p-e_{1}\right\|_{2}
$$

By continuity, there are $\tau=\tau(n)>0$ and $u=u(n)>0$ such that for any two points $p_{1}^{\prime}$ and $p_{2}^{\prime}$ on the unit sphere such that the line passing through $p_{1}^{\prime}$ and $p_{2}^{\prime}$ is at the $\ell_{\infty}^{n}$-distance at most $\tau$ from the origin, we have $\max \left(\left\|p-p_{1}^{\prime}\right\|_{2},\left\|p-p_{2}^{\prime}\right\|_{2}\right) \geq$ $\left\|p-e_{1}\right\|_{2}+u$. Now, for each $\tilde{p} \in \mathbb{S}^{n-1}$ denote by $K_{\tilde{p}}$ the convex cone generated by the vectors $e_{1}, e_{2}, \ldots, e_{n}$ and $\tilde{p}$. It is clear that there is $\kappa=\kappa(n)>0$ such that for any $\tilde{p} \in \mathbb{S}^{n-1}$ with $\|\tilde{p}-p\|_{2} \geq\left\|p-e_{1}\right\|_{2}+u$, we have $\sigma\left(K_{\tilde{p}}, 0\right) \geq(1+\kappa) 2^{-n}$. Using a compactness argument, we infer that a slighly weaker inequality should hold in a small neighborhood of zero, namely, there is $\tau^{\prime}=\tau^{\prime}(n)>0$ such that for any $z \in \tau^{\prime} B_{\infty}^{n}$ and any point $\tilde{p}$ in $\mathbb{S}^{n-1}$ with $\|\tilde{p}-p\|_{2} \geq\left\|p-e_{1}\right\|_{2}+u$ we have that 
the solid angle of $z$ considered as the vertex of the convex cone generated by $e_{i}-z$ $(i=1,2, \ldots, n)$ and $\tilde{p}-z$, is at least $(1+\kappa / 2) 2^{-n}$.

Let us summarize. Let $\ell$ be an affine line in $\mathbb{R}^{n}$ and $z$ be a point on that line such that $z \in \min \left(\tau, \tau^{\prime}\right) B_{\infty}^{n}$ (with $\tau$ and $\tau^{\prime}$ defined above). Further, let $p_{1}$ and $p_{2}$ be intersection points of this line with the unit sphere, and take $p_{j}(j \in\{1,2\})$ having the larger Euclidean distance from $p=(1 / \sqrt{n}, 1 / \sqrt{n}, \ldots, 1 / \sqrt{n})$. Let $K$ be the convex cone with vertex at $z$ generated by the vectors $e_{i}-z(i=1,2, \ldots, n)$ and $p_{j}-z$. Then the solid angle of $z$ (w.r.t. $K$ ) is bounded from below by $(1+\kappa / 2) 2^{-n}$.

The above assertion allows to complete the proof of the claim. Let $x$ and $y$ be two distinct boundary points of a convex body $B \supset B_{\infty}^{n}$, with $x-v, y-v \in$ $\min \left(\tau, \tau^{\prime}\right) B_{\infty}^{n}$ for some vertex $v$ of $B_{\infty}^{n}$. Without loss of generality, we can assume that $v=(-1,-1, \ldots,-1)$. Let $\ell$ be the line passing through $x-v$ and $y-v$, and let $p_{1}, p_{2}$ be intersection points of the line with the unit sphere (let us assume for concreteness that $y-v$ lies in the interval joining $x-v$ and $p_{2}$ ). Note that, since $B$ contains the cube and, in particular, points of the form $v+e_{i}, i=1,2, \ldots, n$, we get that $e_{i}-(x-v), i=1,2, \ldots, n$, lie in the closure of the illuminating cone $\eta(B, x)$, and the same is true for vector $p_{2}-(x-v)$. By analogy, the closure of the cone $\eta(B, y)$ contains the vectors $e_{i}-(y-v)$ and $p_{1}-(y-v)$. Take $p_{j}$ having larger Euclidean distance to $p=(1 / \sqrt{n}, 1 / \sqrt{n}, \ldots, 1 / \sqrt{n})$. If $j=1$ then, by the above reasoning, we have

$$
\begin{aligned}
\sigma(B, y) & \geq \sigma\left(\operatorname{conv}\left\{p_{1}-(y-v), e_{1}-(y-v), \ldots, e_{n}-(y-v)\right\}, y-v\right) \\
& \geq(1+\kappa / 2) 2^{-n} .
\end{aligned}
$$

Similarly, if $j=2$ then $\sigma(B, x) \geq(1+\kappa / 2) 2^{-n}$. The claim follows.

\subsection{Proof of Lemma 3.3}

Claim 4.3 Fix parameters $n>2, \theta \in(0,1)$, assume that $0<\varepsilon \leq \theta / 2$ and $0<\delta \leq$ $\varepsilon / 7$, take a convex body $B$ in a $\star$-position with $\mathrm{d}_{\mathrm{BM}}\left(B, B_{\infty}^{n}\right) \leq 1+\delta$, and pick any set $S \in \mathcal{C}_{n}(\varepsilon, \theta)$. Let $v, v^{\prime}$ be the distinguished pair of vertices with respect to $S$. Then for any $x \in \partial B$ with $x \notin\left(v+9 \delta B_{\infty}^{n}\right) \cup\left(v^{\prime}+9 \delta B_{\infty}^{n}\right), x$ is illuminated by $S$.

Proof Pick any point $x \in \partial B$, and let $w$ be a vertex of the cube $B_{\infty}^{n}$ with the smallest distance to $x$; note that for all non-zero coordinates $x_{j}$ we have $\operatorname{sign}\left(w_{j}\right)=\operatorname{sign}\left(x_{j}\right)$. The choice of $w$ is not unique if $x$ has zero coordinates; in such situation we pick any admissible vertex.

We will consider three cases.

Case 1: $w$ is adjacent neither to $v$, nor to $v^{\prime}$. In this case, $S$ contains an element $s=-w+\varepsilon y$, for some $y \in B_{\infty}^{n}$. By (2), $B \subset(1+2 \delta) B_{\infty}^{n}$, and we get for every $j=1, \ldots, n$, and for any $a \in(0,1 / 2]$ :

$$
\begin{aligned}
\left|(x+a(-w+\varepsilon y))_{j}\right| & \leq\left|x_{j}-a w_{j}\right|+a \varepsilon \leq \max (1+2 \delta-a, a)+a \varepsilon \\
& =1+2 \delta-a+a \varepsilon .
\end{aligned}
$$


Selecting $a:=3 \delta /(1-\varepsilon)$, we obtain that $x+a s$ is in the interior of $B_{\infty}^{n}$. Since $B_{\infty}^{n} \subset B$, it implies that $x+a s \in B \backslash \partial B$, and therefore $x$ is illuminated by $s$.

Case 2: $w$ is adjacent either to $v$ or to $v^{\prime}$, but $w \notin\left\{v, v^{\prime}\right\}$. Let $i$ be the coordinate in which $v_{i}=v_{i}^{\prime} \neq w_{i}$. In this case, $S$ contains an element $s=-w+(1-\theta) w_{i} e_{i}+\varepsilon y$, for some $y \in B_{\infty}^{n}$. As $B \subset(1+2 \delta) B_{\infty}^{n}$, we get for every $j \in[n] \backslash\{i\}$, and for any $a \in(0,1 / 2]$ :

$$
\left|\left(x+a\left(-w+(1-\theta) w_{i} e_{i}+\varepsilon y\right)\right)_{j}\right| \leq 1+2 \delta-a+a \varepsilon .
$$

In addition,

$$
\left|\left(x+a\left(-\theta w_{i} e_{i}+\varepsilon y\right)\right)_{i}\right| \leq 1+2 \delta-a \theta+a \varepsilon .
$$

Selecting $a:=3 \delta /(\theta-\varepsilon)$ and using the assumptions on $\delta$ and $\varepsilon$, we obtain that $x+a s \in B \backslash \partial B$. As before, it means that $x$ is illuminated by $s \in S$.

Case 3: $w$ is either $v$ or $v^{\prime}$. By construction of $\mathcal{C}_{n}(\varepsilon, \theta), S$ contains an element

$$
s \in-\prod_{j=1}^{n}\left(\frac{v_{j}+v_{j}^{\prime}}{2} \cdot[\theta, 1]\right)+\varepsilon B_{\infty}^{n} .
$$

Let $i$ be the coordinate in which $v$ and $v^{\prime}$ differ (note that it is not the same $i$ as in the previous case). Note that $\operatorname{sign}\left(x_{j}\right)=-\operatorname{sign}\left(s_{j}\right)$ for all non-zero coordinates $x_{j}$ with $j \in[n] \backslash\{i\}$. We then get for every $j \in[n] \backslash\{i\}$ and for any $a \in(0,1 / 2]$ :

$$
\left|(x+a s)_{j}\right| \leq 1+2 \delta-a \theta+a \varepsilon .
$$

In addition,

$$
\left|(x+a s)_{i}\right| \leq\left|x_{i}\right|+a \varepsilon .
$$

Selecting $a:=3 \delta /(\theta-\varepsilon)$, we obtain that $x+a s \in B \backslash \partial B$ unless $\left|x_{i}\right| \geq 1-3 \delta$. Thus, $x$ is illuminated in the direction $s$ whenever $\left|x_{i}\right|<1-3 \delta$. Now, assume that $\left|x_{i}\right| \geq 1-3 \delta$; without loss of generality, $\operatorname{sign}\left(x_{i}\right)=\operatorname{sign}\left(v_{i}\right)$. Similarly to Case 2 , we note that $S$ contains directions $s^{k}=-w^{k}+(1-\theta) w_{k}^{k} e_{k}+\varepsilon y^{k}(k \in[n] \backslash\{i\})$, where $y^{k} \in B_{\infty}^{n}$ and for each $k \in[n] \backslash\{i\}, w^{k}$ is the vertex of $\{-1,1\}^{n}$ adjacent to $v$ that differs from $v$ on the $k$-th coordinate. Fix $k \in[n] \backslash\{i\}$. For any $j \in[n] \backslash\{k\}$ we have, just as in Case 2,

$$
\left|\left(x+a\left(-w^{k}+(1-\theta) w_{k}^{k} e_{k}+\varepsilon y\right)\right)_{j}\right| \leq 1+2 \delta-a+a \varepsilon<1,
$$

where the last inequality holds, for example, with $a:=3 \delta /(1-\varepsilon)$. Further, trivially

$$
\left|\left(x+a\left(-w^{k}+(1-\theta) w_{k}^{k} e_{k}+\varepsilon y\right)\right)_{k}\right| \leq\left|x_{k}\right|+a \theta+a \varepsilon,
$$

and, with the last choice of $a$, the quantity is strictly less than 1 whenever $\left|x_{k}\right|<1-9 \delta$. Thus, we get that $x$ is illuminated by one of the $n$ directions $\left\{s, s^{k}, k \in[n] \backslash\{i\}\right\}$, 
whenever there is a coordinate of $x$ which is strictly less than $1-9 \delta$ in absolute value. The result follows.

Claim 4.4 For any $n>2$ and any $\varepsilon \in(0,1)$ there is $\eta^{\prime}=\eta^{\prime}(n, \varepsilon) \in(0,1)$ with the following property. Let $x \in \mathbb{S}^{n-1}$ be a vector such that $x_{j} \geq \varepsilon$ and $x_{k} \leq-\varepsilon$ for some $j \neq k$. Then there is a vector $z \in \mathbb{S}^{n-1}$ with $z_{i} \geq \eta^{\prime}$ for all $i \leq n$, an such that $\langle z, x\rangle=0$.

Proof Denote $a:=\sum_{i \neq j, k} x_{i}$. Let us define a vector $\tilde{z} \in \mathbb{R}^{n}$ by setting $\widetilde{z}_{i}:=1$ for all $i \neq j, k$ and

$$
\widetilde{z}_{j}:=\frac{\sqrt{n}}{x_{j}}>1 ; \quad \widetilde{z}_{k}:=\frac{\sqrt{n}+a}{-x_{k}} .
$$

First, obviously $\langle\widetilde{z}, x\rangle=0$ by the construction. Further, by the Cauchy-Schwarts inequality, $a \geq-\sqrt{n} \sqrt{1-2 \varepsilon^{2}}$ (where we used that $x$ is a unit vector). Thus, all coordinates of $\widetilde{z}$ are greater than $\min \left(1, \sqrt{n}\left(1-\sqrt{1-2 \varepsilon^{2}}\right)\right)$. It remains to choose $z:=\widetilde{z} /\|\tilde{z}\|_{2}$.

The next two claims can be verified with a standard compactness argument.

Claim 4.5 For any $n>2$ and $\kappa \in(0,1)$ there is $\psi^{\prime}=\psi^{\prime}(n, \kappa) \in(0,1)$ with the following property. Let $z^{1}, z^{2}, \ldots, z^{n}$ be vectors in $\mathbb{R}^{n}$ such that $\left\|z^{i}-e_{i}\right\|_{\infty} \leq \psi^{\prime}$. Consider the convex cone

$$
K:=\left\{x \in \mathbb{R}^{n}:\left\langle x, z^{i}\right\rangle \geq 0 \text { for all } i \leq n\right\} .
$$

Then

$$
\sigma(K, 0) \leq(1+\kappa) \cdot 2^{-n}
$$

Claim 4.6 For any $n>2$ and $\eta \in(0,1)$ there is $\psi^{\prime \prime}=\psi^{\prime \prime}(n, \eta) \in(0,1)$ with the following property. Let $\widetilde{z}^{1}, \widetilde{\sim}_{\widetilde{K}}^{2}, \ldots, \widetilde{z}^{n}$ be vectors in $\mathbb{R}^{n}$ such that $\left\|\widetilde{z}^{i}-e_{i}\right\|_{\infty} \leq \psi^{\prime \prime}$. Consider the convex cone $\widetilde{K}$ generated by vectors $\widetilde{z}^{i}(i \leq n)$. Then any unit vector $f \in \mathbb{R}^{n}$ with $f_{i} \geq \eta$ for all $i=1,2, \ldots, n$, lies in the interior of $\widetilde{K}$.

Proof of Lemma 3.3 Fix parameters $n>2$ and $\kappa \in(0,1)$, and let $\eta^{\prime}(\cdot, \cdot), \psi^{\prime}(\cdot, \cdot)$ and $\psi^{\prime \prime}(\cdot, \cdot)$ be as in Claims 4.4, 4.5 and 4.6, respectively. Define $\psi:=\psi^{\prime}(n, \kappa) /(4 n)$ and $\theta_{3.3}:=\psi / 8$. Now, take any $\theta \in\left(0, \theta_{3.3}\right]$, and let

$$
\varepsilon=\varepsilon_{3.3}:=\min \left(1 / n^{2}, \theta \psi / 4\right), \quad \delta=\delta_{3.3}:=\min \left(\psi^{\prime \prime}\left(n, \eta^{\prime}(n, \theta \psi /(8 n))\right) / 4.5, \varepsilon / 7\right) .
$$

Further, take any element $S \in \mathcal{C}_{n}(\varepsilon, \theta)$ and a convex body $B$ in a $\star$-position, with $B_{\infty}^{n} \subset B \subset(1+\delta) B_{\infty}^{n}$. Our goal is to show that for any point $x$ on the boundary of $B$ not illuminated by $S$ we have $\sigma(B, x) \leq(1+\kappa) \cdot 2^{-n}$.

Let $v, v^{\prime}$ be distinguished vertices of the standard cube $B_{\infty}^{n}$ with respect to $S$, and assume that there is a point $x \in \partial B$ which is not illuminated by $S$. By Claim 4.3, 
$x \in\left(v+9 \delta B_{\infty}^{n}\right) \cup\left(v^{\prime}+9 \delta B_{\infty}^{n}\right)$. Without loss of generality, let us assume that $\|x-v\|_{\infty} \leq 9 \delta$ and, moreover, $v=(-1,-1, \ldots,-1)$ and $v^{\prime}=(1,-1,-1, \ldots,-1)$. For $i \leq n$, let $w^{i}$ be the vertex of $B_{\infty}^{n}$ adjacent to $v$, with $w_{i}^{i} \neq v_{i}$. Then $w^{i}-x$ belongs to $\overline{\eta(B, x)}$. By our assumption, $w^{i}-x=2 e_{i}+9 \delta \widetilde{y}^{i}$ for some $\tilde{y}^{i} \in B_{\infty}^{n}$, so that

$$
\overline{\eta(B, x)} \text { contains a convex cone generated by } e_{i}+4.5 \delta \widetilde{y}^{i}, \quad i \leq n \text {. }
$$

Next, we use the assumption that $x$ is not illuminated by any of the directions from $S$. As $x$ is adjacent to a distinguished vertex, there are two types of illuminating directions from $S$ we will consider. First, for any vertex $w$ of $B_{\infty}^{n}$ adjacent to $v$ but distinct from $v^{\prime}, S$ contains a direction of the form

$$
s^{i}:=-\sum_{j \neq i} w_{j} e_{j}-\theta w_{i} e_{i}+\varepsilon y^{i},
$$

for some $y^{i} \in B_{\infty}^{n}$, where $i \geq 2$ is the unique index such that $w_{i} \neq v_{i}=v_{i}^{\prime}$. A trivial computation gives

$$
s^{i}=(1,1,1, \ldots, 1)-(1+\theta) e_{i}+\varepsilon y^{i}, \quad i=2,3, \ldots, n .
$$

Further, $S$ contains the distinguished direction $s^{1}$ such that

$$
s^{1} \in-\prod_{j=1}^{n}\left(\frac{v_{j}+v_{j}^{\prime}}{2} \cdot[\theta, 1]\right)+\varepsilon B_{\infty}^{n} .
$$

Since $x$ is not illuminated by any of the $s^{i}$, s, we have $s^{i} \notin \eta(B, x), i=1,2, \ldots, n$. The Hahn-Banach separation theorem implies that for any $i$ there is an affine hyperplane $H_{i}$ passing through $x$ and parallel to $s^{i}$ but not intersecting the interior of $x+\eta(B, x)$. Choose the unit normal vector $z^{i}$ to $H_{i}$ such that $\left\langle z^{i}, e_{i}\right\rangle \geq 0$.

First, consider the directions $s^{i}$ for $i \geq 2$. Since $\left\langle s^{i}, z^{i}\right\rangle=0$ and in view of (5) we have

$$
\sum_{j \neq i} z_{j}^{i}-\theta z_{i}^{i}+\varepsilon\left\langle y^{i}, z^{i}\right\rangle=0 .
$$

Assume that there exist two coordinates $z_{k}^{i}$ and $z_{\ell}^{i}(k \neq \ell)$ of $z^{i}$ such that $\left|z_{k}^{i}\right|,\left|z_{\ell}^{i}\right| \geq \psi$. Let us suppose for concreteness that $k \neq i$. Then $\left|\sum_{j \neq i} z_{j}^{i}\right| \leq \theta+\varepsilon \sqrt{n} \leq \psi / 4$ and $\left|z_{k}^{i}\right| \geq \psi$, whence there is $u \neq k$ such that $\left|z_{u}^{i}\right| \geq \psi /(2 n)$ and the sign of $z_{u}^{i}$ is opposite to the sign of $z_{k}^{i}$. Then, by Claim 4.4 , there is a vector $f \in \mathbb{S}^{n-1}$ such that $\left\langle f, z^{i}\right\rangle=0$ and $f_{p} \geq \eta^{\prime}(n, \psi /(2 n))$ for all $p \leq n$. By Claim 4.6, applied to the convex cone $\widetilde{K}$ generated by vectors $\widetilde{z}^{i}:=e_{i}+4.5 \delta \widetilde{y}^{i}$, and our choice of parameters, we get that $f$ must belong to the interior of $\widetilde{K}$. On the other hand, the vectors $e_{i}+4.5 \delta \widetilde{y}^{i}$ all belong to the closure of the cone $\eta(B, x)$ (see (4)). Hence, $f \in \eta(B, x)$, so that $H_{i}$ intersects the interior of $x+\eta(B, x)$ and we come to contradiction. Thus, the vector $z^{i}$ has only one 
coordinate $z_{b}^{i}(b \leq n)$ with $\left|z_{b}^{i}\right| \geq \psi$ (note that automatically, $\left.\left|z_{b}^{i}\right|>1-n \psi\right)$. By the choice of $\psi$ and $\theta$, we necessarily have $b=i$, and so $\left\|z^{i}-e_{i}\right\|_{\infty} \leq n \psi<\psi^{\prime}(n, \kappa)$.

Next, we apply a similar argument to direction $s^{1}$. We have $\sum_{j=2}^{n} s_{j}^{1} z_{j}^{1}+s_{1}^{1} z_{1}^{1}=0$, where $\theta-\varepsilon \leq s_{j}^{1} \leq 1+\varepsilon$ for all $j \geq 2$ and $-\varepsilon \leq s_{1}^{1} \leq \varepsilon$. Suppose that for some index $k \geq 2$ we have $\left|z_{k}^{1}\right| \geq \psi$. Since $\left|s_{1}^{1} z_{1}^{1}\right| \leq \varepsilon \leq \theta \psi / 4$, we have $\left|\sum_{j=2}^{n} s_{j}^{1} z_{j}^{1}\right| \leq \theta \psi / 4$ while $\left|s_{k}^{1} z_{k}^{1}\right| \geq \theta \psi / 2$. Hence, there is $\ell \geq 2(\ell \neq k)$ such that $\left|s_{\ell}^{1} z_{\ell}^{1}\right| \geq \theta \psi /(4 n)$, and the sign of $z_{\ell}^{1}$ is opposite to the sign of $z_{k}^{1}$. By Claim 4.4, there is a vector $f \in \mathbb{S}^{n-1}$ such that $\left\langle f, z^{i}\right\rangle=0$ and $f_{p} \geq \eta^{\prime}(n, \theta \psi /(8 n))$ for all $p \leq n$. An application of Claim 4.6 identical to the previous case, yields a contradiction. Thus, $\left|z_{j}^{1}\right| \leq \psi$ for all $j \geq 2$, and $\left\|z^{1}-e_{1}\right\|_{\infty} \leq n \psi<\psi^{\prime}(n, \kappa)$.

Observe that the cone $\eta(B, x)$ is contained inside the set $\left\{y \in \mathbb{R}^{n}:\left\langle y, z^{i}\right\rangle \geq\right.$ 0 \}. Finally, applying Claim 4.5, we get from the last observation and our choice of parameters that $\sigma(B, x) \leq(1+\kappa) \cdot 2^{-n}$, completing the proof.

Remark 4.7 We would like to point out that the proof of Lemma 3.3 requires that $\theta \gg \varepsilon$, and, this is the only place in the proof where this relation is used.

\subsection{Proof of Lemma 3.4}

The assumptions of the lemma imply that there exists a point $x=\left(x_{1}, \ldots, x_{n}\right) \in$ $\operatorname{Int}(B)$ with $\left|x_{i}\right|>1$ for at least one $i \in\{1, \ldots, n\}$. Without loss of generality, we can assume that $x_{1}>1$. Then, by convexity of $B$ and the condition $B_{\infty}^{n} \subset B$, there exists a point $y=\left(y_{1}, 0,0, \ldots, 0\right) \in \operatorname{Int}(B)$ with $y_{1}>1$. Take two vertices $v:=(1,1, \ldots, 1)$ and $v^{\prime}:=(-1,1, \ldots, 1)$ of the unit cube, and define

$$
p:=-\frac{v+v^{\prime}}{2}+\varepsilon^{\prime} e_{1}=\left(\varepsilon^{\prime},-1,-1, \ldots,-1\right),
$$

where $\varepsilon^{\prime}:=\min \left(\varepsilon,\left(y_{1}-1\right) / 2\right)$. Note that $p$ illuminates $v^{\prime}$ as a vertex of the cube, and hence it illuminates $v^{\prime}$ viewed as a boundary point of $B$. Additionally, as $v+p \in \operatorname{Int}(B)$, we have that $p$ illuminates $v$ as a boundary point of $B$.

Consider the collection of directions $S$ consisting of $p$, of all the directions $-w$ where $w$ are the vertices of the unit cube not adjacent to $\left\{v, v^{\prime}\right\}$, and of the directions of the form $-\sum_{j \neq i} w_{j} e_{j}-\theta w_{i} e_{i}$, for the vertices $w$ adjacent to either $v$ or $v^{\prime}$ and different from $v, v^{\prime}$ in $i$-th coordinate $(i=2,3, \ldots, n)$. Note that $S$ belongs to the class $\mathcal{C}_{n}(\varepsilon, \theta)$ and that each point of $\{-1,1\}^{n}$ is illuminated by $S$.

\subsection{Proof of the Lemma 3.6}

We begin with the following elementary claim.

Claim 4.8 Consider the collection $\left\{w^{i}\right\}_{i=1}^{m}$ of $m$ vertices of the discrete cube $\{-1,1\}^{m}$ in $\mathbb{R}^{m}$, where for each $i=1, \ldots, m$, all coordinates of $w^{i}$ except for the $i$-th are +1 , and the $i$-th coordinate is -1 . Then the $(m-1)$-dimensional affine linear span of $w^{1}, w^{2}, \ldots, w^{m}$ contains no other vertices of the cube except $w^{1}, \ldots, w^{m}$. 
Proof Let $x$ be a point in the affine linear span of $w^{1}, \ldots, w^{m}$, i.e., $x=\sum \alpha_{i} w^{i}$ for some coefficients $\alpha_{i} \in \mathbb{R}$ so that $\sum \alpha_{i}=1$. By the definition of $w^{i}$, s, we have $x=\left(1-2 \alpha_{1}, 1-2 \alpha_{2}, \ldots, 1-2 \alpha_{m}\right)$. Assume that $x \in\{-1,1\}^{m}$. Then $\left|1-2 \alpha_{i}\right|=1$ for all $i \leq m$, whence all $\alpha_{i}$ 's are equal to either 0 or 1 . As their sum is one, that means that all the $\alpha_{i}$ 's except for one are equal to zero. Hence, $x$ coincides with one of the $w^{i}$,s, which proves the claim.

Remark 4.9 The above claim implies that for any point $w \in\{-1,1\}^{m} \backslash\left\{w^{1}, \ldots, w^{m}\right\}$ the simplex $\Delta_{w}$ with vertices $\left\{w, w^{1}, w^{2}, \ldots, w^{m}\right\}$ is non-degenerate. In particular, it follows that there is a real number $u=u(m)>0$ depending only on $m$ such that for all $w \in\{-1,1\}^{m} \backslash\left\{w^{1}, \ldots, w^{m}\right\}$ the average $\frac{1}{m+1}\left(w+w^{1}+\cdots+w^{m}\right)$ is at the Euclidean distance at least $u$ from any supporting hyperplane for $\Delta_{w}$.

Let $0<r \leq 1 / 4$ be a parameter, and let $P$ be a convex polytope in $\mathbb{R}^{n}$ such that

$P$ has $2^{n}$ vertices, and $\forall v \in\{-1,1\}^{n} \exists$ a vertex $\widetilde{v}$ of $P$ with $\widetilde{v}-v \in r B_{\infty}^{n}$.

Let us make an immediate elementary observation that will be useful later:

Claim 4.10 For any $n>2$ and $\theta \in(0,1)$ there is $\widetilde{r}=\widetilde{r}(n, \theta) \in(0,1 / 4]$ with the following property. Let $P$ be a convex polytope in $\mathbb{R}^{n}$ satisfying (6) with parameter $r \leq \widetilde{r}$, and let $w$ be any vertex of $P$. Then for any $k \leq n$ the vector $(1-\theta) w_{k} e_{k}$ belongs to $\operatorname{Int}(P)$.

Let $P$ be as in (6). For any $i \leq n$, let $V_{i}^{+}=V_{i}^{+}(P)$ (resp., $V_{i}^{-}=V_{i}^{-}(P)$ ) be the set of vertices of $P$ with positive (resp., negative) $i$-th coordinates. Further, for any $i \leq n$ we introduce special collections $\mathcal{W}_{i}^{+}=\mathcal{W}_{i}^{+}(P) \subset V_{i}^{+}$and $\mathcal{W}_{i}^{-}=\mathcal{W}_{i}^{-}(P) \subset V_{i}^{-}$, where $\mathcal{W}_{i}^{+}$is the set of $n-1$ vertices of $P$ (from $V_{i}^{+}$) each having exactly $n-1$ positive coordinates, and $\mathcal{W}_{i}^{-}$is the set of $n-1$ vertices of $P$ having exactly two negative coordinates (one of them the $i$-th). Note that, when $P$ is the standard cube $[-1,1]^{n}$, the sets $\mathcal{W}_{i}^{+}$and $\mathcal{W}_{i}^{-}$, with the $i$-th components of the vertices removed, directly correspond to the vertex sets from Claim 4.8, with $m=n-1$.

The next statement obviously holds for the standard cube (see Remark 4.9). Its extension for very small perturbations of the cube follows by continuity. We omit the proof.

Claim 4.11 For each $n \geq 3$ there is $r^{\prime \prime}=r^{\prime \prime}(n) \in(0,1 / 4]$ with the following property: Let $P$ be a polytope in $\mathbb{R}^{n}$ satisfying (6) with parameter $r \leq r^{\prime \prime}$, let $i \leq n$, and let $w^{+} \in V_{i}^{+} \backslash \mathcal{W}_{i}^{+}$and $w^{-} \in V_{i}^{-} \backslash \mathcal{W}_{i}^{-}$be two points with $\operatorname{sign}\left(w_{j}^{+}\right)=\operatorname{sign}\left(w_{j}^{-}\right)$for all $j \neq i$. Denote by $H$ the affine linear span of $\mathcal{W}_{i}^{-} \cup\left\{w^{-}\right\}$. Further, let $\widetilde{w}^{+} \in \mathcal{W}_{i}^{+} \cup\left\{w^{+}\right\}$ and $\tilde{w}^{-} \in \mathcal{W}_{i}^{-} \cup\left\{w^{-}\right\}$be two points with $\operatorname{sign}\left(\tilde{w}_{j}^{+}\right)=\operatorname{sign}\left(\widetilde{w}_{j}^{-}\right)$for all $j \neq i$. Set $c^{+}:=\frac{1}{n}\left(w^{+}+\sum_{w \in \mathcal{W}_{i}^{+}} w\right)$ and let $q$ be the point in $H$ such that $q-c^{+}$is parallel to $\widetilde{w}^{-}-\widetilde{w}^{+}$. Then necessarily $q$ belongs to the interior of the simplex $\operatorname{conv}\left(W_{i}^{-} \cup\left\{w^{-}\right\}\right)$.

In the next claim, we give a sufficient condition for $P$ to be a parallelotope. 
Claim 4.12 Suppose that a polytope $P$ in $\mathbb{R}^{n}$ satisfies (6) with $r \leq r^{\prime \prime}$, where $r^{\prime \prime}$ is given by Claim 4.11. Further, suppose that for every $i \leq n$ and every pair of vertices $\left(v^{+}, v^{-}\right) \in\left(V_{i}^{+} \backslash \mathcal{W}_{i}^{+}\right) \times\left(V_{i}^{-} \backslash \mathcal{W}_{i}^{-}\right)$such that $\operatorname{sign}\left(v_{j}^{+}\right)=\operatorname{sign}\left(v_{j}^{-}\right)$for all $j \neq i$, we have that the affine spans of $\mathcal{W}_{i}^{+} \cup\left\{v^{+}\right\}$and $\mathcal{W}_{i}^{-} \cup\left\{v^{-}\right\}$are parallel and, moreover, both spans are supporting hyperplanes for $P$. Then necessarily $P$ is a parallelotope.

Proof Fix any $i \leq n$, let $\left(v^{+}, v^{-}\right)$be a pair as above, and let $z \in \mathbb{S}^{n-1}$ be the (unique) vector such that for some number $\alpha<0$ we have $\langle z, w\rangle=\alpha$ for all $w \in \mathcal{W}_{i}^{-} \cup\left\{v^{-}\right\}$. By the assumption of the claim, the affine spans $H_{1}$ and $H_{2}$ of $\mathcal{W}_{i}^{+} \cup\left\{v^{+}\right\}$and $\mathcal{W}_{i}^{-} \cup\left\{v^{-}\right\}$are parallel, hence there is a number $\beta>0$ such that $\langle z, w\rangle=\beta$ for all $w \in \mathcal{W}_{i}^{+} \cup\left\{v^{+}\right\}$. Next, choose any vertex $w^{+} \in V^{+} \backslash\left(\mathcal{W}_{i}^{+} \cup\left\{v^{+}\right\}\right)$and the vertex $w^{-} \in V^{-} \backslash\left(\mathcal{W}_{i}^{-} \cup\left\{v^{-}\right\}\right)$such that $\operatorname{sign}\left(w_{j}^{+}\right)=\operatorname{sign}\left(w_{j}^{-}\right)$for all $j \neq i$. Again, the affine spans $H_{1}^{\prime}$ and $H_{2}^{\prime}$ of $\mathcal{W}_{i}^{+} \cup\left\{w^{+}\right\}$and $\mathcal{W}_{i}^{-} \cup\left\{w^{-}\right\}$are parallel. On the other hand, by the fact that $H_{1}, H_{2}$ are both supporting hyperplanes for $P$, we get that $\left\langle z, w^{+}\right\rangle \leq \beta$ and $\left\langle z, w^{-}\right\rangle \geq \alpha$.

Assume for a moment that at least one of the last two inequalities is strict. Note that in this case necessarily both inequalities are strict, i.e., $\left\langle z, w^{+}\right\rangle\left\langle\beta\right.$ and $\left\langle z, w^{-}\right\rangle>\alpha$ (otherwise, we would get an immediate contradiction to the fact that $H_{1}$ and $H_{2}$, as well as $H_{1}^{\prime}$ and $H_{2}^{\prime}$ are parallel). Next, let $c^{+}$and $c^{-}$be arithmetic means of points in $\mathcal{W}_{i}^{+} \cup\left\{w^{+}\right\}$and $\mathcal{W}_{i}^{-} \cup\left\{w^{-}\right\}$, respectively, fix any pair $\left(\widetilde{w}^{+}, \widetilde{w}^{-}\right) \in \mathcal{W}_{i}^{+} \times \mathcal{W}_{i}^{-}$ such that $\operatorname{sign}\left(\widetilde{w}_{j}^{+}\right)=\operatorname{sign}\left(\widetilde{w}_{j}^{-}\right)$for all $j \neq i$, and define $q$ as the point in $H_{2}^{\prime}$ such that $q-c^{+}$is parallel to $\widetilde{w}^{-}-\widetilde{w}^{+}$. By Claim 4.11 , the point $q$ belongs to the interior of the simplex $\operatorname{conv}\left(\mathcal{W}_{i}^{-} \cup\left\{w^{-}\right\}\right)$. This gives $\langle z, q\rangle>\alpha$. Let us summarize: we have obtained four points $\widetilde{w}^{+}, c^{+}, q, \widetilde{w}^{-}$forming a parallelogram, but

$$
\begin{aligned}
& \left\langle z, \widetilde{w}^{+}\right\rangle=\beta \geq\left\langle z, c^{+}\right\rangle, \\
& \left\langle z, \widetilde{w}^{-}\right\rangle=\alpha<\langle z, q\rangle,
\end{aligned}
$$

which is impossible. Thus, necessarily $\left\langle z, w^{+}\right\rangle=\beta$ and $\left\langle z, w^{-}\right\rangle=\alpha$, implying that $H_{1}$ coincides with $H_{1}^{\prime}$ and $H_{2}$ coincides with $H_{2}^{\prime}$.

Repeating the above argument for all vertices in $V_{i}^{+} \backslash \mathcal{W}_{i}^{+}$and $V_{i}^{-} \backslash \mathcal{W}_{i}^{-}$, we get that there is a single facet of $P$ containing all vertices $V_{i}^{+}$, and the same holds for $V_{i}^{-}$. Since this condition holds for any $i \leq n$, we get that $P$ is generated by $n$ pairs of parallel hyperplanes, so $P$ is a parallelotope.

Proof of Lemma 3.6 Fix parameters $n>2$ and $\varepsilon>0$, and take $\theta_{3.6}:=\frac{1}{4 n}$. Let $0<$ $\theta \leq \theta_{3.6}$, and let $P$ be a polytope in $\mathbb{R}^{n}$ satisfying condition (6) for $r=r_{3.6}:=$ $\min \left(\widetilde{r}, r^{\prime \prime}, \theta / 5, \varepsilon / 4\right)$, where $\widetilde{r}, r^{\prime \prime}$ are given by Claims 4.10 and 4.11. Assume further that $\mathrm{d}_{\mathrm{BM}}\left(P, B_{\infty}^{n}\right) \neq 1$. We want to show that $P$ can be illuminated by some $S \in$ $\mathcal{C}_{n}(\varepsilon, \theta)$. By Claim 4.12, there is $i \leq n$ and two sets of vertices $\mathcal{W}_{i}^{+} \cup\left\{v^{+}\right\}$and $\mathcal{W}_{i}^{-} \cup\left\{v^{-}\right\}\left(\right.$where $\operatorname{sign}\left(v_{j}^{+}\right)=\operatorname{sign}\left(v_{j}^{-}\right)$for all $\left.j \neq i\right)$, such that one of the following two conditions holds.

Case 1. The affine span of $\mathcal{W}_{i}^{+} \cup\left\{v^{+}\right\}$is parallel to the affine span of $\mathcal{W}_{i}^{-} \cup\left\{v^{-}\right\}$, but at least one of the spans is not a supporting hyperplane for $P$. Without loss of generality, assume that the simplex with vertices $\mathcal{W}_{i}^{+} \cup\left\{v^{+}\right\}$is not a part of a facet, 
whence the point $c^{+}:=\frac{1}{n}\left(v^{+}+\sum_{w \in \mathcal{W}_{i}^{+}} w\right)$ belongs to the interior of $P$. Consider the unit vector $z$ orthogonal to both affine spans of $\mathcal{W}_{i}^{+} \cup\left\{v^{+}\right\}$and $\mathcal{W}_{i}^{-} \cup\left\{v^{-}\right\}$, chosen so that $\left\langle z, v^{-}\right\rangle\left\langle\left\langle z, v^{+}\right\rangle\right.$. Then, for all sufficiently small $\xi>0$, the vector $p=c^{+}-v^{+}+\xi z$ illuminates both $v^{+}$and $v^{-}$. Indeed, illumination of $v^{+}$is obvious. As for $v^{-}$, note that, by Claim 4.11, there is a point $q$ in the interior of the $(n-1)$ $\operatorname{simplex} \operatorname{conv}\left(\mathcal{W}_{i}^{-} \cup\left\{v^{-}\right\}\right)$such that $q-c^{+}$is parallel to $v^{-}-v^{+}$. This means that $q-v^{-}=c^{+}-v^{+}$, whence $v^{-}+p$ belongs to the interior of $P$ for small enough $\xi$.

We construct the illuminating set $\mathcal{S}$ for $P$ as follows. Take any vertex $w$ of $P$ and let $\widetilde{w}$ be the corresponding vertex of $B_{\infty}^{n}$ (i.e., such that $w-\widetilde{w} \in r B_{\infty}^{n}$ ). Further, denote by $v$ and $v^{\prime}$ the vertices of $B_{\infty}^{n}$ corresponding to $v^{+}$and $v^{-}$. If $\widetilde{w}$ is not adjacent to $\left\{v, v^{\prime}\right\}$ then we add to $\mathcal{S}$ the direction $-w$ (observe that $-w \in-\widetilde{w}+\varepsilon B_{\infty}^{n}$ and that $-w$ illuminates $w$ ). Next, if $\widetilde{w}$ is adjacent to $v$ or $v^{\prime}$ but does not belong to $\left\{v, v^{\prime}\right\}$ then we pick the direction $-w+(1-\theta) w_{k} e_{k}$, where $k \neq i$ is the unique index such that $v_{k}=v_{k}^{\prime} \neq \widetilde{w}_{k}$. Observe that, in view of Claim 4.10, the direction $-w+(1-\theta) w_{k} e_{k}$ illuminates $w$. On the other hand, $-w+(1-\theta) w_{k} e_{k} \in-\widetilde{w}+(1-\theta) \widetilde{w}_{k} e_{k}+\varepsilon B_{\infty}^{n}$. Finally, if $\widetilde{w}$ coincides with either $v$ or $v^{\prime}$ then we consider the direction $p$ constructed above. Observe that $\left|p_{i}\right| \leq 3 r$ (assuming $\xi$ is small). Further, for any $j \neq i$ we have $\left|c_{j}^{+}-v_{j}^{+}+\xi z_{j}\right| \geq \frac{1}{n}-2 r-\xi \geq 2 \theta$ and $\operatorname{sign}\left(p_{j}\right)=\operatorname{sign}\left(c_{j}^{+}-v_{j}^{+}+\xi z_{j}\right)=-\operatorname{sign}\left(v_{j}\right)$, where we used the definition of the set $\mathcal{W}_{i}^{+}$and the assumption that $\xi$ is small. Hence,

$$
\frac{1}{2} p \in-\prod_{j=1}^{n}\left(\frac{v_{j}+v_{j}^{\prime}}{2} \cdot[\theta, 1]\right)+2 r B_{\infty}^{n} .
$$

We add $p / 2$ to $S$ as the distinguished direction. By the construction of $S$, it illuminates all vertices of $P$, and belongs to the class $\mathcal{C}_{n}(\varepsilon, \theta)$.

Case 2. Suppose that the affine span of $\mathcal{W}_{i}^{+} \cup\left\{v^{+}\right\}$is not parallel to the affine span of $\mathcal{W}_{i}^{-} \cup\left\{v^{-}\right\}$. Choose a vertex $\widetilde{v}^{+} \in \mathcal{W}_{i}^{+} \cup\left\{v^{+}\right\}$with the smallest distance to the affine span $H$ of $\mathcal{W}_{i}^{-} \cup\left\{v^{-}\right\}$, and fix the unit vector $z$ orthogonal to $H$ and such that $\left\langle z, \widetilde{v}^{+}\right\rangle>0$. Let $\widetilde{v}^{-}$be the vertex in $\mathcal{W}_{i}^{-} \cup\left\{v^{-}\right\}$corresponding to $\widetilde{v}^{+}$(i.e., $\operatorname{sign}\left(\widetilde{v}_{j}^{+}\right)=\operatorname{sign}\left(\tilde{v}_{j}^{-}\right)$for all $\left.j \neq i\right)$, let $c^{+}$be the average of points in $\mathcal{W}_{i}^{+} \cup\left\{v^{+}\right\}$, and consider the vector $\tilde{p}:=c^{+}-\widetilde{v}^{+}-\xi z$, where $\xi>0$ is a small parameter. Observe that for small $\xi, \widetilde{p}$ illuminates $\widetilde{v}^{+}$by construction. Further, by Claim 4.11 , there is a point $q$ in the interior of the simplex $\mathcal{W}_{i}^{-} \cup\left\{v^{-}\right\}$, such that $q-c^{+}$is parallel to $\widetilde{v}^{-}-\widetilde{v}^{+}$. Since the span of $\mathcal{W}_{i}^{+} \cup\left\{v^{+}\right\}$is not parallel to $H$ and by our choice of $\widetilde{v}^{+}$and $z$, we have that $\left\langle c^{+}-\widetilde{v}^{+}, z\right\rangle>0$ while $\left\langle q-\widetilde{v}^{-}, z\right\rangle=0$. Hence, the point $\widetilde{v}^{-}+\left(c^{+}-\widetilde{v}^{+}\right)$ lies in the interior of the interval joining $c^{+}$and $q$ i.e., in the interior of $P$, and so $\tilde{p}$ illuminates $\widetilde{v}^{-}$provided that $\xi$ is sufficiently small. We will construct an illuminating set $S$ for $P$ taking $\widetilde{/ 2}$ as the distinguished direction. The rest of the argument is very similar to the first case, and we omit the details.

Acknowledgements The first author is supported by an AMS-Simons travel Grant. The second author is partially supported by the Simons Foundation. The work was partially supported by the National Science Foundation under Grant No. DMS-1440140 and the Viterbi postdoctoral fellowship while the authors were in residence at the Mathematical Sciences Research Institute in Berkeley, California, during the Fall 2017 semester. Both authors would like to thank the Referee for valuable suggestions. 
Open Access This article is distributed under the terms of the Creative Commons Attribution 4.0 International License (http://creativecommons.org/licenses/by/4.0/), which permits unrestricted use, distribution, and reproduction in any medium, provided you give appropriate credit to the original author(s) and the source, provide a link to the Creative Commons license, and indicate if changes were made.

\section{References}

1. Bezdek, K.: Classical Topics in Discrete Geometry. CMS Books in Mathematics/Ouvrages de Mathématiques de la SMC. Springer, New York (2010)

2. Bezdek, K.: Illuminating spindle convex bodies and minimizing the volume of spherical sets of constant width. Discrete Comput. Geom. 47(2), 275-287 (2012)

3. Bezdek, K., Bisztriczky, T.: A proof of Hadwiger's covering conjecture for dual cyclic polytopes. Geom. Dedicata 68(1), 29-41 (1997)

4. Bezdek, K., Khan, M.A.: The geometry of homothetic covering and illumination. In: Conder, M.D.E., Deza, A., Ivić Weiss, A. (eds.) Discrete Geometry and Symmetry. Springer Proceedings in Mathematics \& Statistics, vol. 234, pp. 1-30. Springer, Cham (2018)

5. Boltyanskiĭ, V.G.: Solution of the illumination problem for belt-bodies. Math. Notes 58(3-4), 10291032 (1996)

6. Boltyanski, V., Martini, H., Soltan, P.S.: Excursions into Combinatorial Geometry. Universitext. Springer, Berlin (1997)

7. Bourgain, J., Milman, V.D.: New volume ratio properties for convex symmetric bodies in $R^{n}$. Invent. Math. 88(2), 319-340 (1987)

8. Hadwiger, H.: Ungelöste probleme, Nr. 20. Elem. Math. 12, 121 (1957)

9. Hadwiger, H.: Ungelöste probleme. Nr. 38. Elem. Math. 15, 130-131 (1960)

10. Iriyeh, H., Shibata, M.: Symmetric Mahler's conjecture for the volume product in the three dimensional case (2017). arXiv:1706.01749

11. Kuperberg, G.: From the Mahler conjecture to Gauss linking integrals. Geom. Funct. Anal. 18(3), 870-892 (2008)

12. Lassak, M.: Solution of Hadwiger's covering problem for centrally symmetric convex bodies in $E^{3}$. J. Lond. Math. Soc. II 30(3), 501-511 (1984)

13. Levi, F.W.: Überdeckung eines Eibereiches durch Parallelverschiebungen seines offenen Kerns. Arch. Math. 6(5), 369-370 (1955)

14. Livshyts, G., Tikhomirov, K.: Randomized coverings of a convex body with its homothetic copies, and illumination (2016). arXiv:1606.08876

15. Martini, H.: Some results and problems around zonotopes. In: Böröczky, K., Fejes Tóth, G. (eds.) Intuitive Geometry (Siófok, 1985). Colloquia Mathematica Societatis János Bolyai, vol. 48, pp. 383418. North-Holland, Amsterdam (1987)

16. Naszódi, M.: A spiky ball. Mathematika 62(2), 630-636 (2016)

17. Nazarov, F., Petrov, F., Ryabogin, D., Zvavitch, A.: A remark on the Mahler conjecture: local minimality of the unit cube. Duke Math. J. 154(3), 419-430 (2010)

18. Rogers, C.A.: A note on coverings. Mathematika 4, 1-6 (1957)

19. Rogers, C.A., Shephard, G.C.: The difference body of a convex body. Arch. Math. 8, 220-233 (1957)

20. Schramm, O.: Illuminating sets of constant width. Mathematika 35(2), 180-189 (1988)

21. Tikhomirov, K.: Illumination of convex bodies with many symmetries. Mathematika 63(2), 372-382 (2017)

22. Zong, C.: A quantitative program for Hadwigers covering conjecture. Sci. China Math. 53(9), 25512560 (2010)

Publisher's Note Springer Nature remains neutral with regard to jurisdictional claims in published maps and institutional affiliations. 